\title{
Minimizing Depth of Decision Trees with Hypotheses
}

\author{
Mohammad Azad 10000-0001-9851-1420], Igor Chikalov2 [0000-0002-1010-6605], \\ Shahid Hussain ${ }^{3}[0000-0002-1698-2809]$, and Mikhail \\ Moshkov 4 [0000-0003-0085-9483] \\ 1 Jouf University \\ College of Computer and Information Sciences \\ Department of Computer Science \\ Sakaka 72441, Saudi Arabia \\ mmazad@ju.edu.sa \\ 2 Intel Corporation, 5000 W Chandler Blvd, Chandler, AZ 85226, USA \\ igor.chikalov@gmail.com \\ 3 Habib University \\ Dhanani School of Science and Engineering \\ Computer Science Program \\ Karachi 75290, Pakistan \\ hussain.shahid@gmail.com \\ 4 King Abdullah University of Science and Technology (KAUST) \\ Computer, Electrical and Mathematical Sciences \& Engineering Division \\ Thuwal 23955-6900, Saudi Arabia \\ mikhail.moshkov@kaust.edu.sa
}

\begin{abstract}
In this paper, we consider decision trees that use both conventional queries based on one attribute each and queries based on hypotheses about values of all attributes. Such decision trees are similar to ones studied in exact learning, where membership and equivalence queries are allowed. We present dynamic programming algorithms for minimization of the depth of above decision trees and discuss results of computer experiments on various data sets and randomly generated Boolean functions.
\end{abstract}

Keywords: Decision tree $\cdot$ Hypothesis $\cdot$ Depth.

\section{Introduction}

Decision trees are widely used in many areas of computer science and related fields, for example, test theory (initiated by Chegis and Yablonskii [6]), rough set theory (initiated by Pawlak [8-10]), and exact learning (initiated by Angluin $[4,5])$. These theories are closely related. In particular, attributes from rough set theory and test theory correspond to membership queries from exact learning. Exact learning considers additionally the so-called equivalence queries. The notion of "minimally adequate teacher" that allows both membership and equivalence queries was discussed by Angluin in [3]. Relations between exact learning 
and PAC learning proposed by Valiant [11] were discussed in [4]. In this paper, we add the notion of a hypothesis to the model that has been considered in rough set theory as well in test theory. This model allows us to use an analog of equivalence queries.

Let $T$ be a decision table with $n$ conditional attributes $f_{1}, \ldots, f_{n}$ having values from the set $\omega=\{0,1,2, \ldots\}$ in which rows are pairwise different and each row is labeled with a decision from $\omega$. For a given row of $T$, we should recognize the decision attached to this row. To this end, we can use decision trees based on two types of queries. We can ask about the value of an attribute $f_{i} \in\left\{f_{1}, \ldots, f_{n}\right\}$ on the given row. We will obtain an answer of the kind $f_{i}=\delta$, where $\delta$ is the number in the intersection of the given row and the column $f_{i}$. We can also ask if a hypothesis $f_{1}=\delta_{1}, \ldots, f_{n}=\delta_{n}$ is true, where $\delta_{1}, \ldots, \delta_{n}$ are numbers from the columns $f_{1}, \ldots, f_{n}$, respectively. Either this hypothesis will be confirmed or we obtain a counterexample in the form $f_{i}=\sigma$, where $f_{i} \in\left\{f_{1}, \ldots, f_{n}\right\}$ and $\sigma$ is a number from the column $f_{i}$ different from $\delta_{i}$. The considered hypothesis is called proper if $\left(\delta_{1}, \ldots, \delta_{n}\right)$ is a row of the table $T$. We consider the depth of a decision tree as its time complexity, which is equal to the maximum number of queries in a path from the root to a terminal node of the tree.

Decision trees using hypotheses can be essentially more efficient than the decision trees using only attributes. Let us consider an example, the problem of computation of the conjunction $x_{1} \wedge \cdots \wedge x_{n}$. The minimum depth of a decision tree solving this problem using the attributes $x_{1}, \ldots, x_{n}$ is equal to $n$. However, the minimum depth of a decision tree solving this problem using proper hypotheses is equal to 1 : it is enough to ask only about the hypothesis $x_{1}=1, \ldots, x_{n}=1$. If it is true, then the considered conjunction is equal to 1 . Otherwise, it is equal to 0 .

We consider the following five types of decision trees:

1. Decision trees that use only attributes.

2. Decision trees that use only hypotheses.

3. Decision trees that use both attributes and hypotheses.

4. Decision trees that use only proper hypotheses.

5. Decision trees that use both attributes and proper hypotheses.

For each type of decision trees, we design a dynamic programming algorithm that, for a given decision table, finds the minimum depth of a decision tree of the considered type for this table. Note that algorithms for the minimization of the depth for decision trees of type 1 were considered in [1] for decision tables with one-valued decisions and in [2] for decision tables with many-valued decisions.

For the conjunction of $n$ variables, the considered algorithms construct a decision tree of type 1 with the depth equal to $n$ and the decision trees of types $2,3,4$, and 5 with the depth equal to 1 .

It is interesting to study not only specially chosen examples as the conjunction of $n$ variables. We compute the minimum depth of a decision tree for each of the considered five types for eight decision tables from the UCI ML Repository 
[7]. We do the same for randomly generated Boolean functions with $n$ variables, where $n=3, \ldots, 6$.

In particular, from the results obtained for Boolean functions it follows that, in general case, the decision trees of types 2 and 4 are better than the decision trees of type 1 , and the decision trees of types 3 and 5 are better than the decision trees of types 2 and 4 .

The rest of the paper is organized as follows. In Sects. 2 and 3, we consider main notions. In Sects. 4 and 5 - dynamic programming algorithms for the depth minimization. Section 6 contains results of computer experiments and Sect. 7 short conclusions.

\section{Decision Tables}

A decision table is a rectangular table $T$ with $n \geq 1$ columns filled with numbers from the set $\omega=\{0,1,2, \ldots\}$ of nonnegative integers. Columns of this table are labeled with the conditional attributes $f_{1}, \ldots, f_{n}$. Rows of the table are pairwise different. Each row is labeled with a number from $\omega$ that is interpreted as a decision. Rows of the table are interpreted as tuples of values of the conditional attributes.

A decision table can be represented by a word over the alphabet $\{0,1, ;, \mid\}$ in which numbers from $\omega$ are in binary representation (are represented by words over the alphabet $\{0,1\}$ ), the symbol ";" is used to separate two numbers from $\omega$, and the symbol "|" is used to separate two rows (we add to each row corresponding decision as the last number in the row). The length of this word will be called the size of the decision table.

A decision table $T$ is called empty if it has no rows. The table $T$ is called degenerate if it is empty or all rows of $T$ are labeled with the same decision.

We denote $F(T)=\left\{f_{1}, \ldots, f_{n}\right\}$ and denote by $D(T)$ the set of decisions attached to the rows of $T$. For any conditional attribute $f_{i} \in F(T)$, we denote by $E\left(T, f_{i}\right)$ the set of values of the attribute $f_{i}$ in the table $T$. We denote by $E(T)$ the set of conditional attributes of $T$ for which $\left|E\left(T, f_{i}\right)\right| \geq 2$.

A system of equations over $T$ is an arbitrary equation system of the kind

$$
\left\{f_{i_{1}}=\delta_{1}, \ldots, f_{i_{m}}=\delta_{m}\right\},
$$

where $m \in \omega, f_{i_{1}}, \ldots, f_{i_{m}} \in F(T)$, and $\delta_{1} \in E\left(T, f_{i_{1}}\right), \ldots, \delta_{m} \in E\left(T, f_{i_{m}}\right)$ (if $m=0$, then the considered equation system is empty).

Let $T$ be a nonempty table. A subtable of $T$ is a table obtained from $T$ by removal of some rows. We correspond to each equation system $S$ over $T$ a subtable $T S$ of the table $T$. If the system $S$ is empty, then $T S=T$. Let $S$ be nonempty and $S=\left\{f_{i_{1}}=\delta_{1}, \ldots, f_{i_{m}}=\delta_{m}\right\}$. Then $T S$ is the subtable of the table $T$ containing the rows from $T$, which in the intersection with the columns $f_{i_{1}}, \ldots, f_{i_{m}}$ have numbers $\delta_{1}, \ldots, \delta_{m}$, respectively. Such nonempty subtables, including the table $T$, are called separable subtables of $T$. We denote by $S E P(T)$ the set of separable subtables of the table $T$. 


\section{Decision Trees}

Let $T$ be a nonempty decision table with $n$ conditional attributes $f_{1}, \ldots, f_{n}$. We consider the decision trees with two types of queries. We can choose an attribute $f_{i} \in F(T)=\left\{f_{1}, \ldots, f_{n}\right\}$ and ask about its value. This query has the following possible answers: $\left\{f_{i}=\delta\right\}$, where $\delta \in E\left(T, f_{i}\right)$. We can formulate a hypothesis over $T$ in the form of $H=\left\{f_{1}=\delta_{1}, \ldots, f_{n}=\delta_{n}\right\}$, where $\delta_{1} \in$ $E\left(T, f_{1}\right), \ldots, \delta_{n} \in E\left(T, f_{n}\right)$, and ask about this hypothesis. This query has the following possible answers: $H,\left\{f_{1}=\sigma_{1}\right\}, \sigma_{1} \in E\left(T, f_{1}\right) \backslash\left\{\delta_{1}\right\}, \ldots,\left\{f_{n}=\sigma_{n}\right\}, \sigma_{n} \in$ $E\left(T, f_{n}\right) \backslash\left\{\delta_{n}\right\}$. The first answer means that the hypothesis is true. Other answers are counterexamples. The hypothesis $H$ is called proper for $T$ if $\left(\delta_{1}, \ldots, \delta_{n}\right)$ is a row of the table $T$.

A decision tree over $T$ is a marked finite directed tree with the root in which

- Each terminal node is labeled with a number from the set $D(T) \cup\{0\}$.

- Each node, which is not terminal (such nodes are called working), is labeled with an attribute from the set $F(T)$ or with a hypothesis over $T$.

- If a working node is labeled with an attribute $f_{i}$ from $F(T)$, then, for each possible answer $\left\{f_{i}(x)=\delta\right\}, \delta \in E\left(T, f_{i}\right)$, there is exactly one edge labeled with this answer, which leave this node and there are no any other edges leaving this node.

- If a working node is labeled with a hypothesis $H=\left\{f_{1}=\delta_{1}, \ldots, f_{n}=\right.$ $\left.\delta_{n}\right\}$ over $T$, then, for each possible answer $H,\left\{f_{1}=\sigma_{1}\right\}, \sigma_{1} \in E\left(T, f_{1}\right) \backslash$ $\left\{\delta_{1}\right\}, \ldots,\left\{f_{n}=\sigma_{n}\right\}, \sigma_{n} \in E\left(T, f_{n}\right) \backslash\left\{\delta_{n}\right\}$, there is exactly one edge labeled with this answer, which leaves this node and there are no any other edges leaving this node.

Let $\Gamma$ be a decision tree over $T$ and $v$ be a node of $\Gamma$. We now define an equation system $S(\Gamma, v)$ over $T$ associated with the node $v$. We denote by $\xi$ the directed path from the root of $\Gamma$ to the node $v$. If there are no working nodes in $\xi$, then $S(\Gamma, v)$ is the empty system. Otherwise, $S(\Gamma, v)$ is the union of equation systems attached to the edges of the path $\xi$.

A decision tree $\Gamma$ over $T$ is called a decision tree for $T$ if, for any node $v$ of $\Gamma$,

- The node $v$ is terminal if and only if the subtable $T S(\Gamma, v)$ is degenerate.

- If $v$ is a terminal node and the subtable $T S(\Gamma, v)$ is empty, then the node $v$ is labeled with the decision 0 .

- If $v$ is a terminal node and the subtable $T S(\Gamma, v)$ is nonempty, then the node $v$ is labeled with the decision attached to all rows of $T S(\Gamma, v)$.

A complete path in $\Gamma$ is an arbitrary directed path from the root to a terminal node in $\Gamma$. As the time complexity of a decision tree, we consider its depth that is the maximum number of working nodes in a complete path in the tree or, which is the same, the maximum length of a complete path in the tree. We denote by $h(\Gamma)$ the depth of a decision tree $\Gamma$.

We will use the following notation: 
- $h^{(1)}(T)$ is the minimum depth of a decision tree for $T$, which uses only attributes from $F(T)$.

$-h^{(2)}(T)$ is the minimum depth of a decision tree for $T$, which uses only hypotheses over $T$.

- $h^{(3)}(T)$ is the minimum depth of a decision tree for $T$, which uses both attributes from $F(T)$ and hypotheses over $T$.

- $h^{(4)}(T)$ is the minimum depth of a decision tree for $T$, which uses only proper hypotheses over $T$.

- $h^{(5)}(T)$ is the minimum depth of a decision tree for $T$, which uses both attributes from $F(T)$ and proper hypotheses over $T$.

\section{Construction of Directed Acyclic Graph $\Delta(T)$}

Let $T$ be a nonempty decision table with $n$ conditional attributes $f_{1}, \ldots, f_{n}$. We now consider an algorithm $\mathcal{A}_{0}$ for the construction of a directed acyclic graph (DAG) $\Delta(T)$, which will be used for the study of decision trees. Nodes of this graph are some separable subtables of the table $T$. During each iteration we process one node. We start with the graph that consists of one node $T$, which is not processed and finish when all nodes of the graph are processed.

Algorithm $\mathcal{A}_{0}$ (construction of DAG $\Delta(T)$ ).

Input: A nonempty decision table $T$ with $n$ conditional attributes $f_{1}, \ldots, f_{n}$. Output: Directed acyclic graph $\Delta(T)$.

1. Construct the graph that consists of one node $T$, which is not marked as processed.

2. If all nodes of the graph are processed, then the algorithm halts and returns the resulting graph as $\Delta(T)$. Otherwise, choose a node (table) $\Theta$ that has not been processed yet.

3. If $\Theta$ is degenerate, then mark the node $\Theta$ as processed and proceed to step 2.

4. If $\Theta$ is not degenerate, then, for each $f_{i} \in E(\Theta)$, draw a bundle of edges from the node $\Theta$. Let $E\left(\Theta, f_{i}\right)=\left\{a_{1}, \ldots, a_{k}\right\}$. Then draw $k$ edges from $\Theta$ and label these edges with systems of equations $\left\{f_{i}=a_{1}\right\}, \ldots,\left\{f_{i}=a_{k}\right\}$. These edges enter nodes $\Theta\left\{f_{i}=a_{1}\right\}, \ldots, \Theta\left\{f_{i}=a_{k}\right\}$, respectively. If some of the nodes $\Theta\left\{f_{i}=a_{1}\right\}, \ldots, \Theta\left\{f_{i}=a_{k}\right\}$ are not present in the graph, then add these nodes to the graph. Mark the node $\Theta$ as processed and return to step 2 .

The following statement about time complexity of the algorithm $\mathcal{A}_{0}$ follows immediately from Proposition 3.3 [1].

Proposition 1. The time complexity of the algorithm $\mathcal{A}_{0}$ is bounded from above by a polynomial on the size of the input table $T$ and the number $|S E P(T)|$ of different separable subtables of $T$. 
In general case, the time complexity of the algorithm $\mathcal{A}_{0}$ is exponential depending on the size of the input decision tables. Note that, in Sect. 3.4 of the book [1], classes of decision tables are described for each of which the number of separable subtables of decision tables from the class is bounded from above by a polynomial on the number of columns in the tables. For each of these classes, the time complexity of the algorithm $\mathcal{A}_{0}$ is polynomial depending on the size of the input decision tables.

\section{Minimizing the Depth of Decision Trees}

Let $T$ be a nonempty decision table with $n$ conditional attributes $f_{1}, \ldots, f_{n}$. We can use the DAG $\Delta(T)$ to compute values $h^{(1)}(T), \ldots, h^{(5)}(T)$. Let $t \in$ $\{1, \ldots, 5\}$. To find the value $h^{(t)}(T)$, for each node $\Theta$ of the DAG $\Delta(T)$, we compute the value $h^{(t)}(\Theta)$. It will be convenient for us to consider not only subtables that are nodes of $\Delta(T)$ but also empty subtable $\Lambda$ of $T$ and subtables $T_{r}$ that contain only one row $r$ of $T$ and are not nodes of $\Delta(T)$. We begin with these special subtables and terminal nodes of $\Delta(T)$ (nodes without leaving edges) that are degenerate separable subtables of $T$ and step-by-step move to the table $T$.

Let $\Theta$ be a terminal node of $\Delta(T)$ or $\Theta=T_{r}$ for some row $r$ of $T$. Then $h^{(t)}(\Theta)=0$ : the decision tree that contains only one node labeled with the decision attached to all rows of $\Theta$ is a decision tree for $\Theta$. If $\Theta=\Lambda$, then $h^{(t)}(\Theta)=0$ : the decision tree that contains only one node labeled with 0 will be considered as a decision tree for $\Lambda$.

Let $\Theta$ be a nonterminal node of $\Delta(T)$ such that, for each child $\Theta^{\prime}$ of $\Theta$, we already know the value $h^{(t)}\left(\Theta^{\prime}\right)$. Based on this information, we can find the minimum depth of a decision tree for $\Theta$, which uses for the subtables corresponding to children of the root decision trees of the type $t$ and in which the root is labeled

- With an attribute from $F(T)$ (we denote by $h_{a}^{(t)}(\Theta)$ the minimum depth of such a decision tree).

- With a hypothesis over $T$ (we denote by $h_{h}^{(t)}(\Theta)$ the minimum depth of such a decision tree).

- With a proper hypothesis over $T$ (we denote by $h_{p}^{(t)}(\Theta)$ the minimum depth of such a decision tree).

Since $\Theta$ is nondegenerate, the set $E(\Theta)$ is nonempty. We now describe three procedures for computing the values $h_{a}^{(t)}(\Theta), h_{h}^{(t)}(\Theta)$, and $h_{p}^{(t)}(\Theta)$, respectively.

Let us consider a decision tree $\Gamma\left(f_{i}\right)$ for $\Theta$ in which the root is labeled with an attribute $f_{i} \in E(\Theta)$. For each $\delta \in E\left(T, f_{i}\right)$, there is an edge that leaves the root and enters a node $v(\delta)$. This edge is labeled with the equation system $\left\{f_{i}=\delta\right\}$. The node $v(\delta)$ is the root of a decision tree of the type $t$ for $\Theta\left\{f_{i}=\delta\right\}$ for which the depth is equal to $h^{(t)}\left(\Theta\left\{f_{i}=\delta\right\}\right)$. It is clear that

$$
h\left(\Gamma\left(f_{i}\right)\right)=1+\max \left\{h^{(t)}\left(\Theta\left\{f_{i}=\delta\right\}\right): \delta \in E\left(T, f_{i}\right)\right\} .
$$


Since $h^{(t)}\left(\Theta\left\{f_{i}=\delta\right\}\right)=h^{(t)}(\Lambda)=0$ for any $\delta \in E\left(T, f_{i}\right) \backslash E\left(\Theta, f_{i}\right)$,

$$
h\left(\Gamma\left(f_{i}\right)\right)=1+\max \left\{h^{(t)}\left(\Theta\left\{f_{i}=\delta\right\}\right): \delta \in E\left(\Theta, f_{i}\right)\right\} .
$$

It is clear that, for any $\delta \in E\left(\Theta, f_{i}\right)$, the subtable $\Theta\left\{f_{i}=\delta\right\}$ is a child of $\Theta$ in the DAG $\Delta(T)$, i.e., we know the value $h^{(t)}\left(\Theta\left\{f_{i}=\delta\right\}\right)$.

One can show that $h\left(\Gamma\left(f_{i}\right)\right)$ is the minimum depth of a decision tree for $\Theta$ in which the root is labeled with the attribute $f_{i}$ and which uses for the subtables corresponding to children of the root decision trees of the type $t$.

We should not consider attributes $f_{i} \in F(T) \backslash E(\Theta)$ since, for each such attribute, there is $\delta \in E\left(T, f_{i}\right)$ with $\Theta\left\{f_{i}=\delta\right\}=\Theta$, i.e., based on this attribute we cannot construct an optimal decision tree for $\Theta$. As a result, we have

$$
h_{a}^{(t)}(\Theta)=\min \left\{h\left(\Gamma\left(f_{i}\right)\right): f_{i} \in E(\Theta)\right\} .
$$

Computation of $h_{a}^{(t)}(\Theta)$. Construct the set of attributes $E(\Theta)$. For each attribute $f_{i} \in E(\Theta)$, compute the value $h\left(\Gamma\left(f_{i}\right)\right)$ using (1). Compute the value $h_{a}^{(t)}(\Theta)$ using $(2)$.

Remark 1. Let $\Theta$ be a nonterminal node of the DAG $\Delta(T)$ such that, for each child $\Theta^{\prime}$ of $\Theta$, we already know the value $h^{(t)}\left(\Theta^{\prime}\right)$. Then the procedure of computation of the value $h_{a}^{(t)}(\Theta)$ has polynomial time complexity depending on the size of decision table $T$.

A hypothesis $H=\left\{f_{1}=\delta_{1}, \ldots, f_{n}=\delta_{n}\right\}$ over $T$ is called admissible for $\Theta$ and an attribute $f_{i} \in F(T)=\left\{f_{1}, \ldots, f_{n}\right\}$ if, for any $\sigma \in E\left(T, f_{i}\right) \backslash\left\{\delta_{i}\right\}$, $\Theta\left\{f_{i}=\sigma\right\} \neq \Theta$. The hypothesis $H$ is not admissible for $\Theta$ and an attribute $f_{i} \in F(T)$ if and only if $\left|E\left(\Theta, f_{i}\right)\right|=1$ and $\delta_{i} \notin E\left(\Theta, f_{i}\right)$. The hypothesis $H$ is called admissible for $\Theta$ if it is admissible for $\Theta$ and any attribute $f_{i} \in F(T)$.

Let us consider a decision tree $\Gamma(H)$ for $\Theta$ in which the root is labeled with an admissible for $\Theta$ hypothesis $H=\left\{f_{1}=\delta_{1}, \ldots, f_{n}=\delta_{n}\right\}$. The set of answers for the query corresponding to the hypothesis $H$ is equal to $A(H)=\left\{H,\left\{f_{1}=\right.\right.$ $\left.\left.\sigma_{1}\right\}, \ldots,\left\{f_{n}=\sigma_{n}\right\}: \sigma_{1} \in E\left(T, f_{1}\right) \backslash\left\{\delta_{1}\right\}, \ldots, \sigma_{n} \in E\left(T, f_{n}\right) \backslash\left\{\delta_{n}\right\}\right\}$. For each $S \in A(H)$, there is an edge that leaves the root of $\Gamma(H)$ and enters a node $v(S)$. This edge is labeled with the equation system $S$. The node $v(S)$ is the root of a decision tree of the type $t$ for $\Theta S$, which depth is equal to $h^{(t)}(\Theta S)$. It is clear that

$$
h(\Gamma(H))=1+\max \left\{h^{(t)}(\Theta S): S \in A(H)\right\} .
$$

We have $\Theta H=\Lambda$ or $\Theta H=T_{r}$ for some row $r$ of $T$. Therefore $h^{(t)}(\Theta H)=0$. Since $H$ is admissible for $\Theta, E\left(\Theta, f_{i}\right) \backslash\left\{\delta_{i}\right\}=\emptyset$ for any attribute $f \in F(T) \backslash E(\Theta)$. It is clear that $\Theta\left\{f_{i}=\sigma\right\}=\Lambda$ and $h^{(t)}\left(\Theta\left\{f_{i}=\sigma\right\}\right)=0$ for any attribute $f_{i} \in E(\Theta)$ and any $\sigma \in E\left(T, f_{i}\right) \backslash\left\{\delta_{i}\right\}$ such that $\sigma \notin E\left(\Theta, f_{i}\right)$. Therefore

$$
h(\Gamma(H))=1+\max \left\{h^{(t)}\left(\Theta\left\{f_{i}=\sigma\right\}\right): f_{i} \in E(\Theta), \sigma \in E\left(\Theta, f_{i}\right) \backslash\left\{\delta_{i}\right\}\right\} .
$$

It is clear that, for any $f_{i} \in E(\Theta)$ and any $\sigma \in E\left(\Theta, f_{i}\right) \backslash\left\{\delta_{i}\right\}$, the subtable $\Theta\left\{f_{i}=\sigma\right\}$ is a child of $\Theta$ in the DAG $\Delta(T)$, i.e., we know the value $h^{(t)}\left(\Theta\left\{f_{i}=\right.\right.$ $\sigma\})$. 
One can show that $h(\Gamma(H))$ is the minimum depth of a decision tree for $\Theta$ in which the root is labeled with the hypothesis $H$ and which uses for the subtables corresponding to children of the root decision trees of the type $t$.

We should not consider hypotheses that are not admissible for $\Theta$ since, for each such hypothesis $H$ for corresponding query, there is an answer $S \in A(H)$ with $\Theta S=\Theta$, i.e., based on this hypothesis we cannot construct an optimal decision tree for $\Theta$.

Computation of $h_{h}^{(t)}(\Theta)$. First, we construct a hypothesis

$$
H_{\Theta}=\left\{f_{1}=\delta_{1}, \ldots, f_{n}=\delta_{n}\right\}
$$

for $\Theta$. Let $f_{i} \in F(T) \backslash E(\Theta)$. Then $\delta_{i}$ is equal to the only number in the set $E\left(\Theta, f_{i}\right)$. Let $f_{i} \in E(\Theta)$. Then $\delta_{i}$ is the minimum number from $E\left(\Theta, f_{i}\right)$ for which $h^{(t)}\left(\Theta\left\{f_{i}=\delta_{i}\right\}\right)=\max \left\{h^{(t)}\left(\Theta\left\{f_{i}=\sigma\right\}\right): \sigma \in E\left(\Theta, f_{i}\right)\right\}$. It is clear that $H_{\Theta}$ is admissible for $\Theta$. Compute the value $h\left(\Gamma\left(H_{\Theta}\right)\right)$ using (3). Simple analysis of (3) shows that $h\left(\Gamma\left(H_{\Theta}\right)\right)=h_{h}^{(t)}(\Theta)$.

Remark 2. Let $\Theta$ be a nonterminal node of the DAG $\Delta(T)$ such that, for each child $\Theta^{\prime}$ of $\Theta$, we already know the value $h^{(t)}\left(\Theta^{\prime}\right)$. Then the procedure of computation of the value $h_{h}^{(t)}(\Theta)$ has polynomial time complexity depending on the size of decision table $T$.

Computation of $h_{p}^{(t)}(\Theta)$. For each row $r=\left(\delta_{1}, \ldots, \delta_{n}\right)$ of the decision table $T$, we check if the corresponding proper hypothesis $H_{r}=\left\{f_{1}=\delta_{1}, \ldots, f_{n}=\delta_{n}\right\}$ is admissible for $\Theta$. For each admissible for $\Theta$ proper hypothesis $H_{r}=\left\{f_{1}=\right.$ $\left.\delta_{1}, \ldots, f_{n}=\delta_{n}\right\}$, we compute the value $h\left(\Gamma\left(H_{r}\right)\right)$ using (3). One can show that the minimum among the obtained numbers is equal to $h_{p}^{(t)}(\Theta)$.

Remark 3. Let $\Theta$ be a nonterminal node of the DAG $\Delta(T)$ such that, for each child $\Theta^{\prime}$ of $\Theta$, we already know the value $h^{(t)}\left(\Theta^{\prime}\right)$. Then the procedure of computation of the value $h_{p}^{(t)}(\Theta)$ has polynomial time complexity depending on the size of decision table $T$.

For $t=1, \ldots, 5$, we describe an algorithm $\mathcal{A}_{t}$ that, for a given decision table $T$, calculates the value $h^{(t)}(T)$, which is the minimum depth of a decision tree of the type $t$ for the table $T$. During the work of this algorithm, we find for each node $\Theta$ of the DAG $\Delta(T)$ the value $h^{(t)}(\Theta)$.

Algorithm $\mathcal{A}_{t}$ (computation of $h^{(t)}(T)$ ).

Input: A nonempty decision table $T$ and the directed acyclic graph $\Delta(T)$.

Output: The value $h^{(t)}(T)$.

1. If a number is attached to each node of the DAG $\Delta(T)$, then return the number attached to the node $T$ as $h^{(t)}(T)$ and halt the algorithm. Otherwise, choose a node $\Theta$ of the graph $\Delta(T)$ without attached number, which is either a terminal node of $\Delta(T)$ or a nonterminal node of $\Delta(T)$ for which all children have attached numbers. 
2. If $\Theta$ is a terminal node, then attach to it the number $h^{(t)}(\Theta)=0$ and proceed to step 1.

3. If $\Theta$ is not a terminal node, then depending on the value $t$ do the following:

- In the case $t=1$, compute the value $h_{a}^{(1)}(\Theta)$ and attach to $\Theta$ the value $h^{(1)}(\Theta)=h_{a}^{(1)}(\Theta)$.

- In the case $t=2$, compute the value $h_{h}^{(2)}(\Theta)$ and attach to $\Theta$ the value $h^{(2)}(\Theta)=h_{h}^{(2)}(\Theta)$.

- In the case $t=3$, compute the values $h_{a}^{(3)}(\Theta)$ and $h_{h}^{(3)}(\Theta)$, and attach to $\Theta$ the value $h^{(3)}(\Theta)=\min \left\{h_{a}^{(3)}(\Theta), h_{h}^{(3)}(\Theta)\right\}$.

- In the case $t=4$, compute the value $h_{p}^{(4)}(\Theta)$ and attach to $\Theta$ the value $h^{(4)}(\Theta)=h_{p}^{(4)}(\Theta)$.

- In the case $t=5$, compute the values $h_{a}^{(5)}(\Theta)$ and $h_{p}^{(5)}(\Theta)$, and attach to $\Theta$ the value $h^{(5)}(\Theta)=\min \left\{h_{a}^{(5)}(\Theta), h_{p}^{(5)}(\Theta)\right\}$.

Proceed to step 1.

Using Remarks 1-3 one can prove the following statement.

Proposition 2. For $t=1, \ldots, 5$, the time complexity of the algorithm $\mathcal{A}_{t}$ is bounded from above by a polynomial on the size of the input table $T$ and the number $|S E P(T)|$ of different separable subtables of $T$.

\section{Results of Experiments}

We make experiments with eight decision tables from the UCI ML Repository [7]. Results are represented in Table 1. The first three columns contain the information about the considered decision table $T$ : its name, the number of rows, and the number of conditional attributes. The last five columns contain values $h^{(1)}(T), \ldots, h^{(5)}(T)$ (minimum values for each decision table are in bold).

Table 1. Experimental results for decision tables from [7]

\begin{tabular}{lrrrrrrr}
\hline $\begin{array}{l}\text { Decision } \\
\text { table } T\end{array}$ & $\begin{array}{r}\text { Number of Number of } \\
\text { rows }\end{array}$ & $h^{(1)}(T)$ & $h^{(2)}(T)$ & $h^{(3)}(T)$ & $h^{(4)}(T)$ & $h^{(5)}(T)$ \\
\hline BALANCE-SCALE & 625 & 5 & $\mathbf{4}$ & $\mathbf{4}$ & $\mathbf{4}$ & $\mathbf{4}$ & $\mathbf{4}$ \\
BREAST-CANCER & 266 & 10 & 6 & 6 & $\mathbf{5}$ & 6 & $\mathbf{5}$ \\
CARS & 1728 & 7 & $\mathbf{6}$ & $\mathbf{6}$ & $\mathbf{6}$ & $\mathbf{6}$ & $\mathbf{6}$ \\
HAYES-ROTH-DATA & 69 & 5 & $\mathbf{4}$ & $\mathbf{4}$ & $\mathbf{4}$ & $\mathbf{4}$ & $\mathbf{4}$ \\
NURSERY & 12960 & 9 & 8 & 8 & $\mathbf{7}$ & 8 & $\mathbf{7}$ \\
SOYBEAN-SMALL & 47 & 36 & $\mathbf{2}$ & 4 & $\mathbf{2}$ & 6 & $\mathbf{2}$ \\
TIC-TAC-TOE & 958 & 10 & 6 & 6 & $\mathbf{5}$ & 8 & 6 \\
ZOO-DATA & 59 & 17 & $\mathbf{4}$ & $\mathbf{4}$ & $\mathbf{4}$ & 5 & $\mathbf{4}$ \\
\hline Average & & & 5.00 & 5.25 & 4.63 & 5.88 & 4.75 \\
\hline
\end{tabular}


Decision trees with the minimum depth using attributes (type 1) are optimal for 5 decision tables, using hypotheses (type 2) are optimal for 4 tables, using attributes and hypotheses (type 3) are optimal for 8 tables, using proper hypotheses (type 4) are optimal for 3 tables, using attributes and proper hypotheses (type 5) are optimal for 7 tables.

For the decision table SOYBEAN-SMALL, we must use attributes to construct an optimal decision tree. For this table, it is enough to use only attributes. For the decision tables BREAST-CANCER and NURSERY, we must use both attributes and hypotheses to construct optimal decision trees. For these tables, it is enough to use attributes and proper hypotheses. For the decision table TIC-TAC-TOE, we must use both attributes and hypotheses to construct optimal decision trees. For this table, it is not enough to use attributes and proper hypotheses.

For $n=3, \ldots, 6$, we generate randomly 100 Boolean functions with $n$ variables. We represent each Boolean function with $n$ variables as a decision table with $n$ columns labeled with these variables and with $2^{n}$ rows that are all possible $n$-tuples of values of the variables. Each row is labeled with the decision that is the value of the function on the corresponding $n$-tuple. For each function, using its decision table representation, we find the minimum depth of a decision tree of the type $t$ computing this function, $t=1, \ldots, 5$. For each Boolean function, each hypothesis over the decision table representing it is proper. Therefore, for each Boolean function, $h^{(2)}=h^{(4)}$ and $h^{(3)}=h^{(5)}$.

Results of experiments are represented in Table 2. The first column contains the number of variables in the considered Boolean functions. The last five columns contain information about values $h^{(1)}, \ldots, h^{(5)}$ in the format ${ }_{\text {min }} A v g_{\text {max }}$.

Table 2. Experimental results for Boolean functions

\begin{tabular}{lrrrrr}
\hline $\begin{array}{l}\text { Number of } \\
\text { variables } n\end{array}$ & $h^{(1)}$ & $h^{(2)}$ & $h^{(3)}$ & $h^{(4)}$ & $h^{(5)}$ \\
\hline 3 & & & & & \\
4 & ${ }_{2} 2.8163_{3}$ & ${ }_{1} 2.0612_{3}$ & ${ }_{1} 1.8878_{2}$ & ${ }_{1} 2.0612_{3}$ & $11.8878_{2}$ \\
5 & ${ }_{3} 3.9400_{4}$ & ${ }_{2} 3.0500_{4}$ & $22.9700_{3}$ & ${ }_{2} 3.0500_{4}$ & $22.9700_{3}$ \\
6 & ${ }_{4} 4.9500_{5}$ & ${ }_{4} 4.0800_{5}$ & ${ }_{3} 3.9900_{4}$ & ${ }_{4} 4.0800_{5}$ & $33.9900_{4}$ \\
\hline
\end{tabular}

From the obtained results it follows that, in general case, the decision trees of types 2 and 4 are better than the decision trees of type 1, and the decision trees of types 3 and 5 are better than the decision trees of types 2 and 4 .

\section{Conclusions}

In this paper, we studied modified decision trees that use both queries based on one attribute each and queries based on hypotheses about values of all attributes. We designed dynamic programming algorithms for minimization of the depth of 
such decision trees and considered results of computer experiments. In the future, we are planning to study the number of nodes in the modified decision trees.

\section{Acknowledgments}

Research reported in this publication was supported by King Abdullah University of Science and Technology (KAUST). The authors are greatly indebted to anonymous reviewers for useful comments and suggestions.

\section{References}

1. AbouEisha, H., Amin, T., Chikalov, I., Hussain, S., Moshkov, M.: Extensions of Dynamic Programming for Combinatorial Optimization and Data Mining, Intelligent Systems Reference Library, vol. 146. Springer (2019)

2. Alsolami, F., Azad, M., Chikalov, I., Moshkov, M.: Decision and Inhibitory Trees and Rules for Decision Tables with Many-valued Decisions, Intelligent Systems Reference Library, vol. 156. Springer (2020)

3. Angluin, D.: Learning regular sets from queries and counterexamples. Inf. Comput. 75(2), 87-106 (1987)

4. Angluin, D.: Queries and concept learning. Mach. Learn. 2(4), 319-342 (1988)

5. Angluin, D.: Queries revisited. Theor. Comput. Sci. 313(2), 175-194 (2004)

6. Chegis, I.A., Yablonskii, S.V.: Logical methods of control of work of electric schemes. Trudy Mat. Inst. Steklov (in Russian) 51, 270-360 (1958)

7. Dua, D., Graff, C.: UCI Machine Learning Repository. University of California, Irvine, School of Information and Computer Sciences (2017), http://archive.ics.uci.edu/ml

8. Pawlak, Z.: Rough sets. Int. J. Parallel Program. 11(5), 341-356 (1982)

9. Pawlak, Z.: Rough Sets - Theoretical Aspects of Reasoning about Data, Theory and Decision Library: Series D, vol. 9. Kluwer (1991)

10. Pawlak, Z., Skowron, A.: Rudiments of rough sets. Inf. Sci. 177(1), 3-27 (2007)

11. Valiant, L.G.: A theory of the learnable. Commun. ACM 27(11), 1134-1142 (1984) 